Resistive plate chambers for 2013-2014 muon upgrade in CMS at LHC

This content has been downloaded from IOPscience. Please scroll down to see the full text. 2014 JINST 9 C10033

(http://iopscience.iop.org/1748-0221/9/10/C10033)

View the table of contents for this issue, or go to the journal homepage for more

Download details:

IP Address: 157.193.98.234

This content was downloaded on 11/06/2015 at $14: 03$

Please note that terms and conditions apply. 
$12^{\text {th }}$ Workshop on Resistive Plate Chambers and Related Detectors, 23-28 FEBRUARY 2014,

TSINGHUA UNIVERSITY, BEIJING, CHINA

\section{Resistive plate chambers for 2013-2014 muon upgrade in CMS at LHC}

S. Colafranceschi, ${ }^{b, 1}$ R. Chudasama, ${ }^{a}$ L.M. Pant,${ }^{a}$ A.K. Mohanty, ${ }^{a}$ R. Sehgal, ${ }^{a}$ S.T. Sehgal, ${ }^{a}$ R.G. Thomas, ${ }^{a}$ A. Sharma, ${ }^{b}$ V. Bhandari, ${ }^{c}$ S. Chand, ${ }^{c}$ A. Kumar, ${ }^{c}$ S. Kumar, ${ }^{c}$ A. Singh, ${ }^{c}$ V. Singh,${ }^{c}$ S. Aly, ${ }^{d, e, 2}$ R. Aly, ${ }^{d, e, 2}$ T. Elkafrawy, ${ }^{e, 2}$ A. Ibrahim, ${ }^{e, 2}$ A. Radi, ${ }^{e}, 2$ A. Sayed, ${ }^{e, 2}$ S. Cauwenbergh, ${ }^{f}$ A. Cimmino, ${ }^{f}$ S. Crucy, ${ }^{f}$ A. Fagot, ${ }^{f}$ G. Garcia,${ }^{f}$ D. Poyraz,${ }^{f}$ S. Salva,${ }^{f}$ F. Thyssen,${ }^{f}$ M. Tytgat, ${ }^{f}$ N. Zaganidis, ${ }^{f}$ M. Abbrescia, ${ }^{g}$ M. Franco,${ }^{g}$ P. laselli, ${ }^{g}$ N. Lacalamita,${ }^{g}$ F. Loddo, ${ }^{g}$ M. Maggi, ${ }^{g}$ G. Pugliese, ${ }^{g}$ P. Verwillingen, ${ }^{g}$ S. Buontempo, ${ }^{h}$ F. Cassese, ${ }^{h}$ N. Cavallo, ${ }^{h}$ S. Energico, ${ }^{h}$ F. Fienga, ${ }^{h}$ F. Fabozzi, ${ }^{h}$ O. Iorio, ${ }^{h}$ L. Lista, ${ }^{h}$ G. Passeggio, ${ }^{h}$ P. Paolucci, ${ }^{h}$ A. Braghieri, ${ }^{i}$ A. Freddi, ${ }^{i}$ S. Guelfo Gigli,${ }^{i}$ P. Montagna,${ }^{i}$ C. Riccardi,${ }^{i}$ P. Salvini, ${ }^{i}{ }^{i}$ F. Vercellati,${ }^{i}$ P. Vitulo, ${ }^{i}$ A. Aleksandrov,${ }^{j}$ V. Genchev,${ }^{j}$ P. laydjiev, ${ }^{j}$ M. Rodozov, ${ }^{j}$ S. Stoykova, ${ }^{j}$ G. Sultanov, ${ }^{j}$ M. Vutova, ${ }^{j}$ Y. Choi, ${ }^{k}$ D. Kim, ${ }^{k}$ L. Benussi, ${ }^{l}$ S. Bianco, ${ }^{l}$ L. Passamonti, ${ }^{l}$ D. Piccolo, ${ }^{l}$ D. Pierluigi, ${ }^{l}$ G. Raffone,${ }^{l}$ A. Russo, ${ }^{l}$ G. Saviano, ${ }^{l}$ A. Ahmad, ${ }^{m}$ W. Ahmed, ${ }^{m}$ I. Ali, ${ }^{m}$ M. Asghar,${ }^{m}$ I. Awan, ${ }^{m}$ H. Hoorani, ${ }^{m}$ S. Muhammad, ${ }^{m}$ H. Shahzad,${ }^{m}$ M. Shoaib, ${ }^{m}$ Y. Ban, ${ }^{n}$ J. Cai, ${ }^{n}$ Q. Li, ${ }^{n}$ S. Liu, ${ }^{n}$ S. Qian, ${ }^{n}$ D. Wang, ${ }^{n}$ Z. Xu, ${ }^{n}$ F. Zhang,${ }^{n}$ S. Bernardino, ${ }^{o}$ H. Ibargüen, ${ }^{o}$ I. Pedraza,,${ }^{o}$ I. Bagaturia, ${ }^{p}$ Z. Tsamalaidze, ${ }^{p}$ A. Cabrera, ${ }^{q}$ L. Chaparro, ${ }^{q}$ J.P. Gomez, ${ }^{q}$ B. Gomez, ${ }^{q}$ J.C. Sanabria, ${ }^{q}$ C. Avila, ${ }^{q}$ A. Dimitrov,${ }^{r}$ R. Hadjiiska, ${ }^{r}$ L. Litov, ${ }^{r}$ B. Pavlov,${ }^{r}$ P. Petkov,${ }^{r}$ W. van Doninck ${ }^{s}$ and I. Crotty ${ }^{t}$

${ }^{a} B A R C$, Electronics Division (BARC),

Trombay, Mumbai 400 085, India

${ }^{b}$ Physics Department CERN, CH-1211 Geneva 23, Switzerland

${ }^{c}$ Panjab University, Department of Physics, Chandigarh Mandir 160 014, India

\footnotetext{
${ }^{1}$ Corresponding author

${ }^{2}$ Supported by the European FP7 EENP2 project
} 
${ }^{d}$ Helwan University, Qism Helwan,

Cairo Governorate, Egypt

${ }^{e}$ Academy of Scientific Research and Technology of the Arab Republic of Egypt,

101 Sharia Kasr El-Ain, Cairo, Egypt

${ }^{f}$ Ghent University, Department of Physics and Astronomy,

Proeftuinstraat 86, BE-9000 Ghent, Belgium

${ }^{g}$ Universita e INFN, Sezione di Bari,

Via Orabona 4, IT-70126 Bari, Italy

${ }^{h}$ INFN, Sezione di Napoli, Complesso Univ. Monte S. Angelo,

Via Cintia, IT-80126 Napoli, Italy

${ }^{i}$ Universita e INFN, Sezione di Pavia,

Via Bassi 6, IT-Pavia, Italy

${ }^{j}$ Bulgarian Academy of Sciences, Inst. for Nucl. Res. and Nucl. Energy,

Tzarigradsko shaussee Boulevard 72, BG-1784 Sofia, Bulgaria

${ }^{k}$ Kyungpook National University, Department of Physics,

80 Daehakro, Bukgu, Daego, 702-701, Republic of Korea

${ }^{l}$ INFN, Laboratori Nazionali di Frascati,

PO Box 13, Via Enrico Fermi 40, IT-00044 Frascati, Italy

${ }^{m}$ Islamabad-NCP, Quaid-I-Azam University,

Islamabad-44000 Pakistan

${ }^{n}$ Peking University, Department of Technical Physics,

CN-100 871 Beijing, China

${ }^{o}$ Benemerita Universidad Autònoma de Puebla, Av. San Claudio y 18 sur,

edif. 111 A Ciudad Universitaria, Col. San Manuel, Puebla, Pue. C.P. 72570, Mexico

${ }^{p}$ Tbilisi University,

1 Ilia Chavchavadze Ave, Tbilisi 0179, Georgia

${ }^{q}$ Universidad de Los Andes,

Apartado Aéreo 4976, Carrera 1E, no. 18A 10, CO-Bogotá, Colombia

${ }^{r}$ University of Sofia, Faculty of Physics, Atomic Physics Department,

5, James Bourchier Boulevard, BG-1164 Sofia, Bulgaria

${ }^{s}$ Vrije Universiteit Brussel,

Boulevard de la Plaine 2, 1050 Ixelles, Belgium

${ }^{t}$ University of Wisconsin, Department of Physics,

1150 University Avenue Madison, WI 53706 USA

E-mail: stefano.colafranceschi@cern.ch

ABSTRACT: During 2013 and 2014 (Long Shutdown LS1) the CMS experiment is upgrading the forward region installing a fourth layer of RPC detectors in order to complete and improve the muon system performances in the view of the foreseen high luminosity run of LHC. The new two endcap disks consists of 144 double-gap RPC chambers assembled at three different production sites: CERN, Ghent (Belgium) and BARC (India). The chamber components as well as the final detectors are subjected to full series of tests established in parallel at all the production sites.

All assembly and test operations have been engineered in order to standardize and improve detector production. In this work the complete chamber construction, quality control procedures and preliminary results will be detailed.

KEYWORDS: Muon spectrometers; Gaseous detectors; Trigger detectors 


\section{Contents}

1 Introduction 1

2 Chamber design, production and quality controls 2

3 Detector performance 5

$\begin{array}{lll}4 & \text { Conclusions } & 7\end{array}$

\section{Introduction}

During the LHC (Large Hadron Collider) Long Shutdown (LS1), the Compact Muon Solenoid [1] (CMS) experiment is undergoing a series of upgrades in order to cope with future increased beam luminosity. In particular the muon system is being upgraded with the implementation of a new layer of Resistive Plate Chambers [2] (RPC) detectors in the endcap regions. Before this upgrade only three RPC layers were installed in the relative three existing endcap disks. The presence of a forth disk equipped with an RPC detector station (figure 1), called RE4 (RPC Endcap disk 4), is going to increase the overall robustness of the CMS muon spectrometer while improving the trigger efficiency adopting a three-out-of-four stations majority trigger logic. The RE4 project is carried out by several institutions and countries. The RPC gas gaps are produced in Korea while Pakistan, Italy and Finland are working on the front-end electronics, DAQ and power system. India is producing and testing 200 cooling sets. Bulgaria, Georgia Mexico and Pakistan are responsible for detector assembly and testing. India, Italy and Pakistan are building the chamber services (gas, cooling and cabling). China provides the readout strips and mechanical frame boxes, and

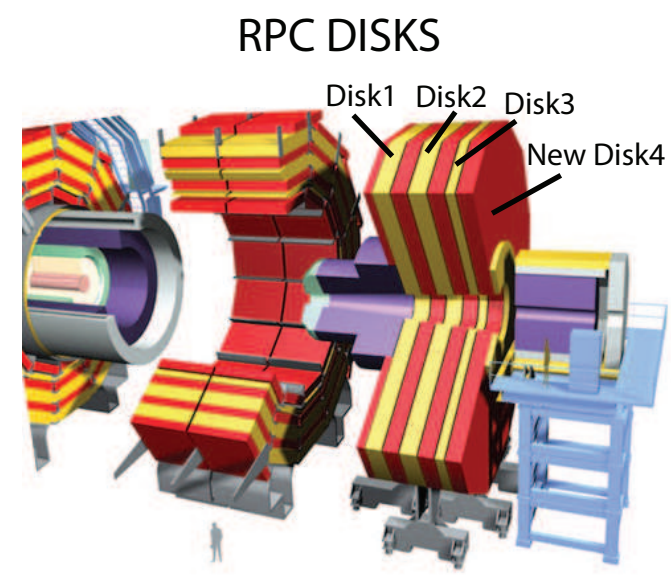

Figure 1. Layout of the existing and the new endcap disks. 


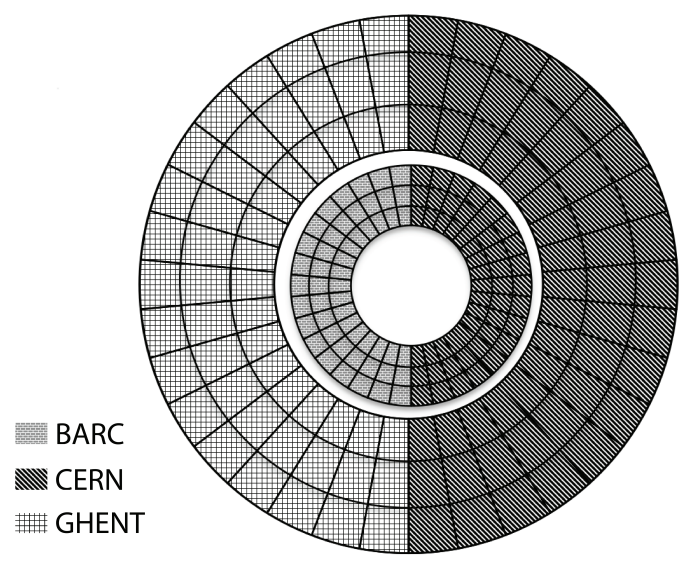

Figure 2. Schematic layout of one of the two new endcap disks.

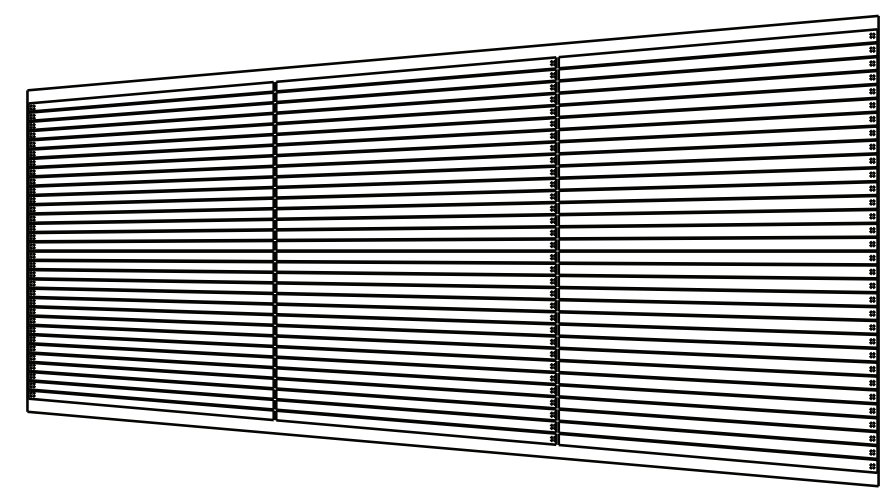

Figure 3. Layout of the readout strips of the RE4 chambers.

participates in the chamber construction and testing at CERN. The RE4 project consists of 72 super-modules, each made of two RPCs, for a total of 144 double-gap RPCs. The RE4 detectors are going to instrument two disks, called RE+ and RE-, located at detector extremities, each disks is made of two rings: the inner ring is called RE4/2 while the outer ring is called RE4/3. In each of the two rings there are 36 trapezoidal shaped detectors, as shown in figure 2, built in three different assembly sites: India (BARC), Belgium (Ghent University) and CERN. BARC and Ghent are in charge of $25 \%$ of the production respectively RE4/2 and RE4/3 while the remaining $50 \%$ of the production is carried by CERN who work on both chamber types RE4/2 and RE4/3.

\section{Chamber design, production and quality controls}

The RE4 project is inheriting the chamber design from the existing RPC Endcap chambers [3].

The detector is made of $2 \mathrm{~mm}$ double-gap RPC, built with $10^{10} \Omega \mathrm{cm}$ HPL electrodes. The top layer of the double gap is segmented in two parts while the bottom layer is made of one gap only, so a chamber is composed of three different gap types. In between the two gap layers a set of readout strips read the $\phi$ coordinate while each strip is segmented in three, along the $\eta$ coordinate (figure 3). 
(a)

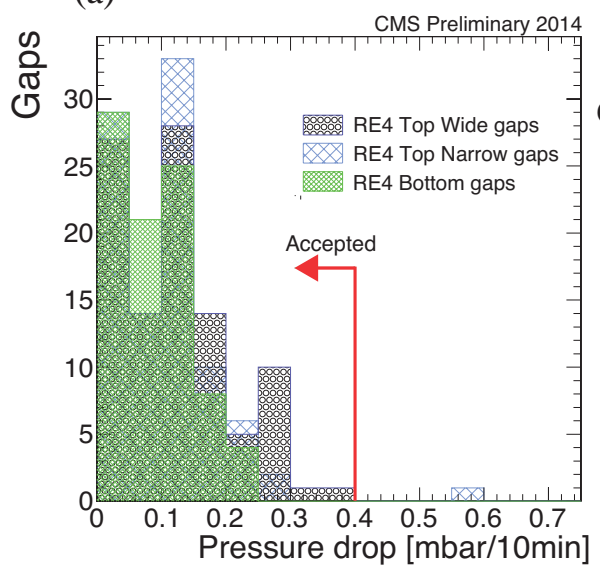

(b)

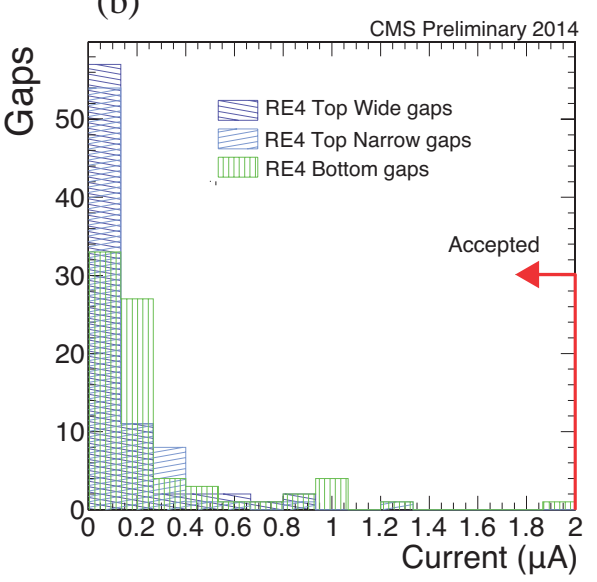

Figure 4. a) Leak rate distributions. b) Dark current and of gaps adopeted for the RE4 detector construction.

The HPL panels $\left(\approx 3500 \mathrm{~m}^{2}\right.$ ) were produced, cut and validated in Italy and shipped to Korea to be assembled to form RPC gaps. The Korean gap manufacturing site [4] is performing several quality control measurements, called Quality Control 1 (QC1), on the HPL panels to ensure the correct resistivity, thickness and roughness and on RPC gaps to monitor the detector performance.

In Korea, the HPL surface quality control is performed after the linseed oil treatment process, to asses the complete and correct polymerization of the oil. The thickness of the insulating plastic spacers was measured to ensure a uniform distance between the RPC gap electrodes. HPL resistivity was evaluated on six RPC gaps irradiated with ${ }^{137}$ Cs source measuring the dark current drawn flushing the gaps with pure argon. Still at the korean gap manifacturing site, preliminary checks are performed to the RPC gaps. To ensure the correct glueing of spacers and HPL panels a gas leak and spacer test is performed. Also gaps are subjected to an high voltage (HV) scan, to measure the dark current using a freon based gas mixture: $95 \% \mathrm{C}_{2} \mathrm{H}_{2} \mathrm{~F}_{4}, 4.5 \% \mathrm{iC}_{4} \mathrm{H}_{10}$. After initial purging of the gap with $201 / \mathrm{h}$ gas flux an high voltage $(1 \mathrm{kV})$ is applied to check for any electrical misconnection. If the measured dark current is lower than $2 \mu \mathrm{A}$ everty 30 minutes the $\mathrm{HV}$ increases by $1 \mathrm{kV}$, while in the region $8-10 \mathrm{kV}$ the increase step is $200 \mathrm{~V}$. Subsequently dark currents are monitored over 96 hours at the operating voltage of $9.7 \mathrm{kV}$. After these first validation tests, a box containing a number of gaps between 30 and 40 is dispatched from Korea to chamber assembly sites.

The second quality control (QC2) pursues the objective of validating the RPC gaps performance repeating some of the test performed in Korea in order to spot any issue that may have occurred during the gap transportation. In this second phase, the three assembly sites perform gap visual inspection, gas tightness, spacer test, and electrical dark current measurement. During the visual inspection each gap is characterized with a detailed checklist which ensures that no any visible damage is present and the gap is eligible to be used. The gas gap spacer and leak tests check that no any gap spacer is detached and leak is within specification ( $0.4 \mathrm{mbar} / 10 \mathrm{minutes})$ as shown in figure $4 \mathrm{a}$.

Once the leak and spacer test are successful, the gaps are subjected to an high voltage scan to measure the dark current response. Figure $4 \mathrm{~b}$ shows the dark current distribution at $10 \mathrm{kV}$. The last test of QC2 concerns the HV scan that aims at measuring the dark current and its stability over a 


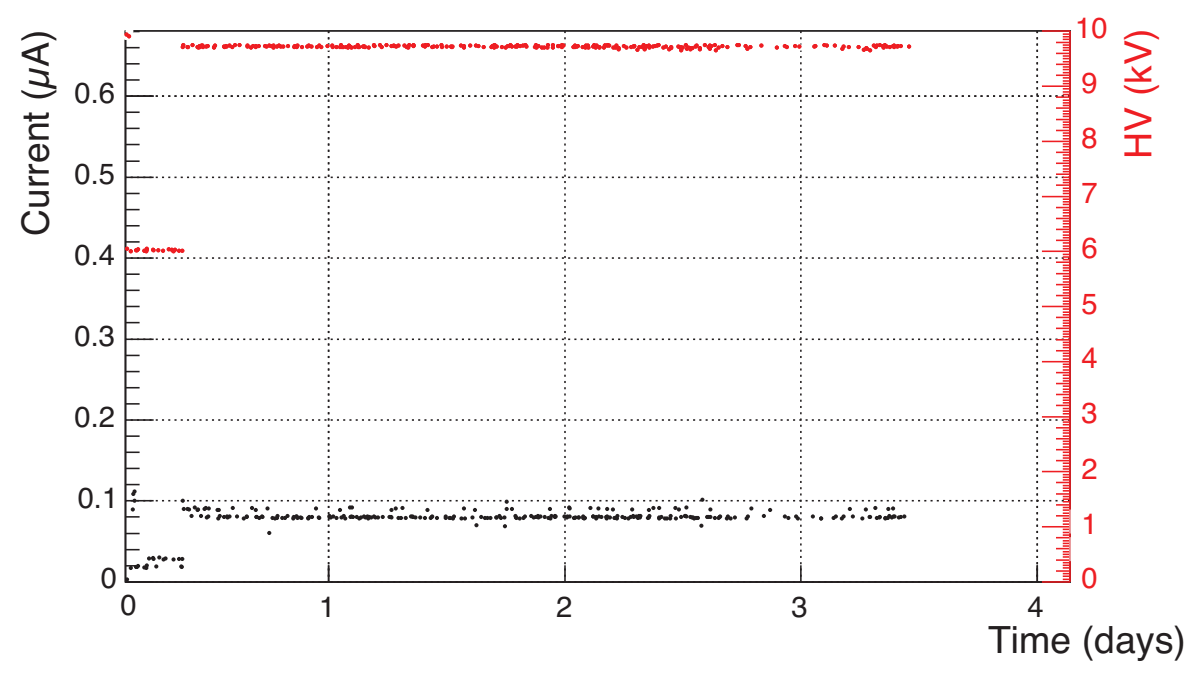

Figure 5. Sample trend of the gap current stability within QC2.

period of 3 days to monitor an eventual drift of the dark current (figure 5).

During the scan the $\mathrm{HV}$ is ramped from $1 \mathrm{kV}$ to $10 \mathrm{kV}$ with steps of $1 \mathrm{kV}$ up to $8 \mathrm{kV}$ and step of $100 \mathrm{~V}$ between $8 \mathrm{kV}$ and $10 \mathrm{kV}$, while during the stability period the $\mathrm{HV}$ is kept at an effective working point of $9.7 \mathrm{kV}$, correcting for pressure and temperature variations. The $\mathrm{HV}$ scan and stability test are performed using the same gas mixture adopted by CMS: $95 \% \mathrm{C}_{2} \mathrm{H}_{2} \mathrm{~F}_{4}$, $4.5 \% \mathrm{iC}_{4} \mathrm{H}_{10}, 0,3 \% \mathrm{SF}_{6}$ with $8000 \mathrm{ppm}$ of water vapor added. Dark current rejection limit was set to $5 \mu \mathrm{A}$. At QC2 an overall rejection factor, due to irreparable damages occured during chamber construction and gap transportation, of $\sim 10 \%$ has been found.

After a set of three gaps is fully validated by assembly sites, the chamber construction and commissioning shall begin. The quality control at the level of the chambers (QC3) foresees: chamber visual inspection, gas tightness, electrical and dark current measurement, cosmic muon commissioning by means of a dedicated cosmic ray telescope. During the visual inspection each chamber undergoes a detailed checklist to validate the manufacturing process of the chamber. Then the leak test measures the chamber gas leak in order to check whether, during chamber manufacturing, the gap gas inlet are correctly connected to chamber service panel. In the electric test the front-end boards are powered and checked to test the connectivity of all channels while the gaps are subjected to an high voltage scan to ensure that the RPC gaps operate without problems.

Finally a chamber by chamber high voltage scan in a cosmic ray telescope concludes the QC3 protocol. This scan aims at characterizing the detector response while measuring main detector performance parameters such as efficiency, cluster size and noise. Since the RE4 chambers are based on double-gap RPCs, each detector is subjected to three independent efficiency scans using three different configuration: double-gap, top single-gap, bottom single-gap. During one efficiency scan, 7 runs at different effective $\mathrm{HV}$ are taken, from 8.5 to $10 \mathrm{kV}$; in each run, $10 \mathrm{k}$ events are collected in approximately 2 hours. The scan is performed using the effective high voltage to correct the applied voltage on each RPC chamber maintaining its gain constant against environmental changes. The applied HV is corrected according to the environmental pressure and temperature 


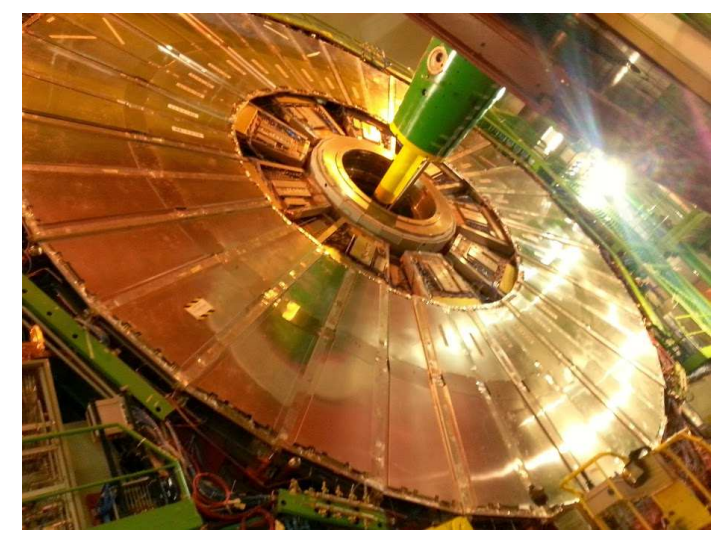

Figure 6. One of the two CMS endcap disks fully instrumented with RPC detectors.

according to eq. (2.1) [5]:

$$
H V_{\mathrm{eff}}=H V \cdot \frac{p_{0}}{p} \frac{T}{T_{0}}
$$

where $\mathrm{p}_{0}=990 \mathrm{mbar}$ and $\mathrm{T}_{0}=293 \mathrm{~K}$.

Each cosmic telescope is equipped with two scintillator layers (top and bottom) that form the trigger by means of a two fold coincidence. Each scintillator layer is segmented in five readout active area with a total surface larger than the detectors under test. In addition to the scintillator coincidence three RPC detectors are added to the trigger for offline selection of muons. The adoption of three RPC detectors allows to remove any fake trigger given by the larger scintillator area, to reject cosmic showers and to reconstruct muon tracks. An offline analysis routine performs a tracking algorithm fitting the position of the fired strips of the three detectors in the 3D space.

The chamber front-end boards are connected via flat cables to TDCs to record the timestamp of cosmic muons firing the detectors under test.

After a successful cosmic test, every chamber is kept powered on and monitored for about three weeks in order to check its stability over time (QC4). After three weeks period, the dark current maximum fluctuation should be less than $50 \%$ for the chamber to be accepted.

To reduce the amount of time needed to install all RE4 detectors at CMS, a pair of chambers, one RE4/2 and one RE4/3 type, is assembled into a super-module (figure 6). Since the RE4/2 and RE4/3 chambers share the same cooling circuit and gas pipes, and are mechanically attached to the same structure, additional commissioning protocols are performed before the real detector installation at CMS. A super-module is subjected to a gas leak test to ensure that the interconnection bewteen the two chambers was properly implemented. The applied overpressure is 5 mbar and the tollerated leak is 0.4 mbar over a period of 10 minutes. A similar leak test is performed on the interconnected water cooling circuits.

\section{Detector performance}

During QC3, assembly sites have the mandate to fully commission each detector. Using the tracking telescope, the performance of RPC detectors under test is evaluated and each chamber fully 
(a)

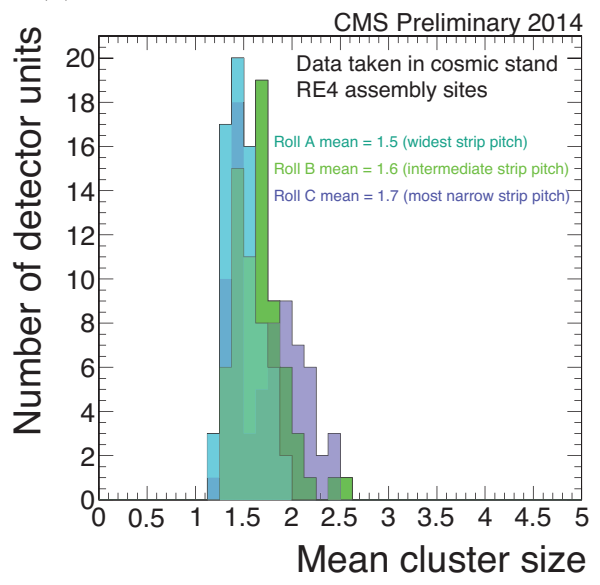

(b)

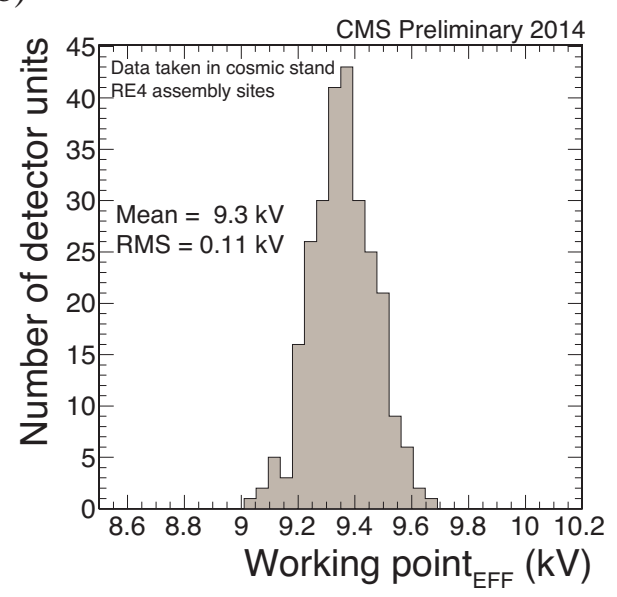

Figure 7. Mean cluster size (a) at the expected nominal HV working point (b) of commissioned RE4 detectors.

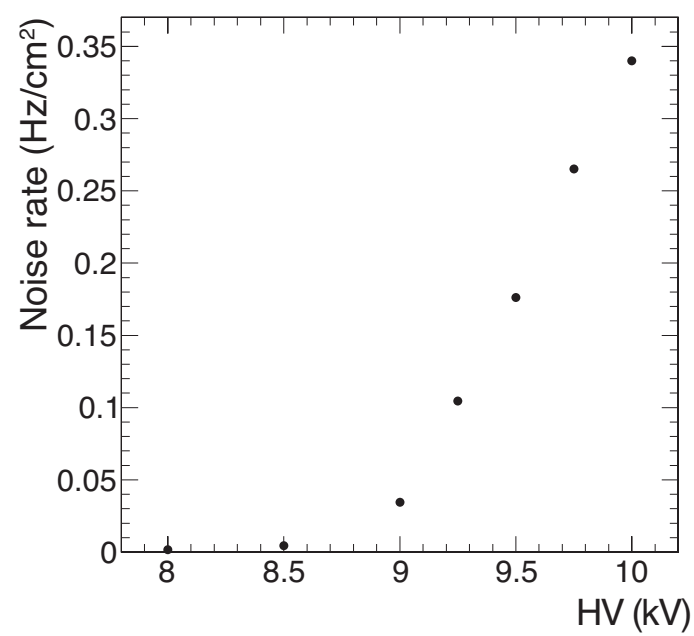

Figure 8. Sample noise rate measured during an HV scan of one detector under test.

characterized. Figure 7a shows the average cluster size distribution evaluated in the three different segments at the expected working point which distribution is shown in figure $7 \mathrm{~b}$. The adopted threshold on the chamber front-end board was set to $210 \mathrm{mV}$ as reference value used at CMS.

The expected working point is defined adding $150 \mathrm{~V}$ to the efficiency at $95 \%$. The eq. (3.1) [6] describes the efficiency along the high voltage scan and it is used to fit the data-points.

$$
\eta=\frac{\epsilon_{\max }}{1+e^{-\lambda\left(H V_{\text {eff }}-H V_{50 \%}\right)}}
$$

The noise rate was automatically measured in all the runs using an additional gate, delayed from the cosmic muon trigger, to the TDCs. Figure 8 shows a sample noise rate measured during an HV scan.

The overall average maximum efficiency in double gap mode $\left(\epsilon_{\max }\right)$ is shown in figure $9 \mathrm{a}$ while the high voltage at $50 \%$ efficiency $\left(\mathrm{HV}_{50 \%}\right)$ is reported in figure $9 \mathrm{~b}$. 
(a)

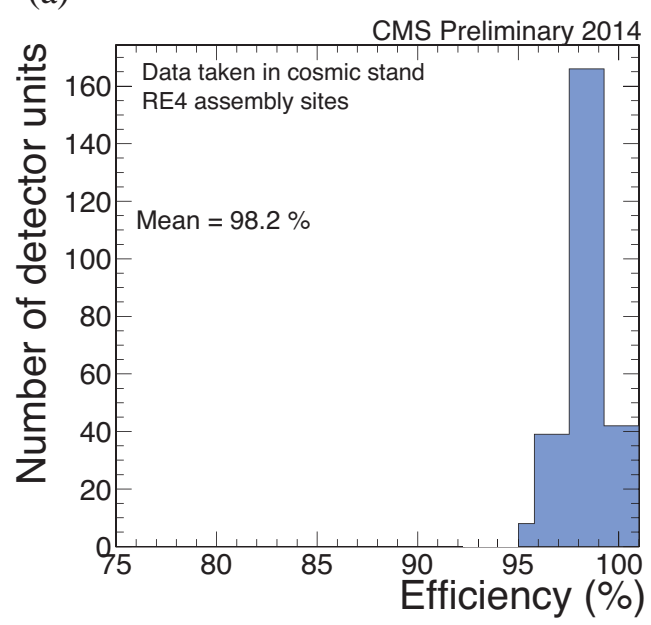

(b)

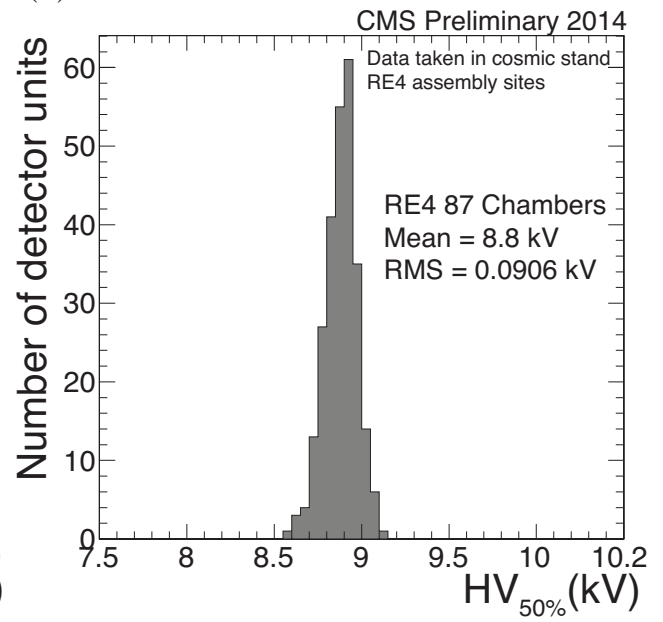

Figure 9. a) Average maximum chamber efficiency (extrapolated from the plateaux curve, see eq. (3.1). b) High voltage distribution at 50\% efficiency (as defined in eq. (3.1).

\section{Conclusions}

RPC collaboration built and commissioned 165 RPC chambers, including spares, in about two years. Today both new disks have been instrumented, commissioned and preliminarly validated during summer 2014 CMS data-taking. The RE4 project succefully upgraded the CMS muon system re-establishing the full production chain of HPL panels, gas gaps, front-end board electronics and RPC detectors. At the level of gap manifacturing a $~ 10 \%$ rejection factor has been found, in agreement with previous production. Compared to the past RPC detector productions, new suppliers produced the detector components using same specification of past productions. Preliminary results from RE4 performance indicate an overall agreement between the new RE4 detectors and the existing CMS RPC disks.

\section{References}

[1] CMS collaboration, The CMS experiment at the CERN LHC, 2008 JINST 3 S08004.

[2] R. Santonico and R. Cardarelli, Development of Resistive Plate Counters, Nucl. Instrum. Meth. 187 (1981) 377.

[3] M. Tytgat et al., The Upgrade of the CMS RPC System during the First LHC Long Shutdown, 2013 JINST 8 T02002 [arXiv: 1209. 1979].

[4] S.K. Park et al., CMS endcap RPC gas gap production for upgrade, 2012 JINST 7 P11013.

[5] M. Abbrescia et al., Resistive plate chambers performances at cosmic rays fluxes, Nucl. Instrum. Meth. A 359 (1995) 603.

[6] M. Abbrescia et al., Cosmic ray tests of double-gap resistive plate chambers for the CMS experiment, Nucl. Instrum. Meth. A 550 (2005) 116. 